\title{
NORMAL TWO-DIMENSIONAL ELLIPTIC SINGULARITIES ${ }^{1}$ \\ BY

\author{
STEPHEN SHING-TOUNG YAU
}

\begin{abstract}
Given a weighted dual graph such that the canonical cycle $K^{\prime}$ exists, is there a singularity corresponding to the given weighted dual graph and which has Gorenstein structure? This is one of the important problems in normal surface singularities. In this paper, we give a necessary and sufficient condition for the existence of Gorenstein structures for weakly elliptic singularities. A necessary and sufficient condition for the existence of maximally elliptic structure is also given. Hence, the above question is answered affirmatively for a special kind of singularities. We also develop a theory for those elliptic Gorenstein singularities with geometric genus equal to three.
\end{abstract}

0. Introduction. Let $p$ be a singularity of a normal two-dimensional analytic space $V$. In [27] Wagreich introduced a definition for $p$ to be weakly elliptic. Weakly elliptic singularities have occurred naturally in papers by Grauert [5], Hirzebruch [9], Laufer [19], Orlik and Wagreich [22], [23], Wagreich [28]. Karras [11], [12] and Saito [24] have studied some of these particular elliptic singularities. Recently Laufer [19] made fundamental progress on the theory of elliptic singularities. He developed a theory for a general class of weakly elliptic singularities which satisfy a minimality condition. These are so-called the minimally elliptic singularities. Choose $V$ to be a Stein space with $p$ as its only singularity. Let $\pi: M \rightarrow V$ be a resolution of $V$. It is known that $\operatorname{dim} H^{1}(M, \vartheta)$ is independent of resolution. Let $v^{\theta_{p}}$ be the germs at $p$ of holomorphic functions on $V$. Minimally elliptic singularities are actually those Gorenstein singularities with $H^{1}(M, \theta)=\mathbf{C}$ [19]. In [30] we develop a theory for a general class of weakly elliptic singularities which satisfy a maximality condition. Maximally elliptic singularities may have $\operatorname{dim} H^{1}(M, \vartheta)$ arbitrarily large. They also include minimally elliptic singularities in the sense of Laufer as a particular case. In [32] we develop a theory for those Gorenstein singularities with $H^{1}(M, \theta)=C^{2}$. The results of this paper are of two kinds.

On the one hand, one might ask the following existence problem. It is known that for Gorenstein singularity, the cycle $K^{\prime}$ exists. Recall that the

Received by the editors December 23, 1976 and, in revised form, January 16, 1978.

AMS (MOS) subject classifications (1970). Primary 13C40, 13C45, 14B05, 14E15, 32C40; Secondary $14 \mathrm{~J} 15,14 \mathrm{C} 20$.

${ }^{1}$ Research supported in part by a National Science Foundation grant. 
cycle $K^{\prime}$ is a negative integral divisor with support on the exceptional set $A$ which is numerically equivalent to canonical divisor. Given a weighted dual graph such that $K^{\prime}$ exists, is there a singularity corresponding to the given weighted dual graph and which has Gorenstein structure? In $\S 1$ we give a necessary and sufficient condition for the existence of Gorenstein structures for weakly elliptic singularities. A necessary and sufficient condition for the existence of maximally elliptic structure is also given. In $\$ 2$ we give a positive answer to the above question for a special kind of singularity.

On the other hand, we develop a theory for those elliptic Gorenstein singularities with $H^{1}(M, \theta)=\mathbf{C}^{3}$. One might use this theory together with the technique which we develop in [31] to get a topological classification of elliptic hypersurface singularities with $H^{1}(M, \theta)=\mathrm{C}^{3}$, i.e. one can list all possible weighted dual graphs which can arise from hypersurface elliptic singularities with $H^{1}(M, \theta)=\mathrm{C}^{3}$.

In this paper $Z$ will denote the fundamental cycle in the sense of M. Artin [1] and $E$ will denote the minimally elliptic cycle in the sense of Laufer [19]. Recall that in [30] we introduce the concept of elliptic sequence which depends only on the topology of the singularities. It turns out that this elliptic sequence plays an important role in elliptic normal singularities. The notation in this paper is standard and can be found in [19] and [30]. Actually, these two papers are good references for the basic knowledge.

We gratefully acknowledge the encouragement and help of Professor Henry B. Laufer during the investigation of these results. We also wish to thank Professors Bennett, Kuga, Siu and Wagreich for discussions of mathematics. Finally, we would like to thank Professors Griffiths, Hironaka and Mumford for their encouragement and their interest in the paper.

1. Preliminaries. Let $\pi: M \rightarrow V$ be a resolution of normal two-dimensional Stein space $V$. We assume that $p$ is the only singularity of $V$. Let $\pi^{-1}(p)=A$ $=\cup_{i} A_{i}, 1 \leqslant i \leqslant n$, be the decomposition of the exceptional set $A$ into irreducible components. Suppose $\pi$ is the minimal good resolution. The topological nature of this embedding of $A$ in $M$ is described by the weighted dual graph $\Gamma$ [10], [15]. The vertices of $\Gamma$ correspond to the $A_{i}$. The edge of $\Gamma$ connecting the vertices corresponding to $A_{i}$ and $A_{j}, i \neq j$, correspond to the points of $A_{i} \cap A_{j}$. Finally, associated to each $A_{i}$ is its genus, $g_{i}$, as a Riemann surface, and its weight, $A_{i} \cdot A_{i}$, the topological self-intersection number. $\Gamma$ will denote the graph, along with the genera and the weights.

A cycle (or divisorial cycle) $D$ on $A$ is an integral combination of the $A_{i}$. $D=\sum d_{i} A_{i}, 1 \leqslant i \leqslant n$ with $d_{i}$ an integer. Let $\theta$ be the sheaf of germs of holomorphic functions on $M$. Let $\theta(-D)$ be the sheaf of germs of holomorphic functions on $M$ which vanish to order $d_{i}$ on $A_{i}$. Let $\mathcal{O}_{D}$ denote $\vartheta / \vartheta(-D)$. We use "dim" to denote dimension over $\mathbf{C}$ 


$$
\chi(D)=\operatorname{dim} H^{0}\left(M, \theta_{D}\right)-\operatorname{dim} H^{1}\left(M, \theta_{D}\right) .
$$

Some authors work instead with the arithmetic genus $P_{a}(D)=1-\chi(D)$. The Riemann-Roch Theorem says

$$
\chi(D)=-\frac{1}{2}(D \cdot D+D \cdot K) .
$$

It follows immediately from (1.2) that if $B$ and $C$ are cycles then

$$
\chi(B+C)=\chi(B)+\chi(C)-B \cdot C .
$$

In (1.2), $K$ is the canonical divisor on $M . D \cdot K$ may be defined as follows. Let $\omega$ be a meromorphic 2-form on $M$ i.e. a meromorphic section of $K$. Let $(\omega)$ be the divisor of $\omega$. Then $D \cdot K=D \cdot(\omega)$ and this number is independent of the choice of $\omega$. In fact, let $g_{i}$ be the geometric genus of $A_{i}$, i.e. the genus of the desingularization of $A_{i}$. Then

$$
A_{i} \cdot K=-A_{i} \cdot A_{i}+2 g_{i}-2+2 \delta_{i}
$$

where $\delta_{i}$ is the "number" of nodes and cusps on $A_{i}$. Each singular point on $A_{i}$ other than a node or cusp counts as at least two nodes. Fortunately, such more complicated singularities will not occur in this paper.

Associated to $\pi$ is a unique fundamental cycle [1, pp. 131-132] such that $Z>0, A_{i} \cdot Z \leqslant 0$ all $A_{i}$, and such that $Z$ is minimal with respect to those two properties. $Z$ may be computed from the intersection matrix as follows [16, p. 607] via what is called a computation sequence (in the sense of Laufer) for $Z$.

$$
\begin{aligned}
Z_{0}=0, \quad Z_{1}=A_{i_{1}}, \quad Z_{2}=Z_{1}+A_{i_{2}}, \ldots \\
Z_{j}=Z_{j-1}+A_{i_{j}} \cdots Z_{l}=Z_{l-1}+A_{i_{l}}
\end{aligned}
$$

where $A_{i_{1}}$ is arbitrary and $A_{i_{j}} \cdot Z_{j-1}>0,1<j \leqslant l . \theta\left(-Z_{j-1}\right) / \theta\left(-Z_{j}\right)$ represents the sheaf of germs of sections of a line bundle over $A_{i_{j}}$ of Chern class $-A_{i_{j}} \cdot Z_{j-1}$. So $H^{0}\left(M, \theta\left(-Z_{j-1}\right) / \theta\left(-Z_{j}\right)\right)=0$ for $j>1$.

$$
0 \rightarrow \vartheta\left(-Z_{j-1}\right) / \vartheta\left(-Z_{j}\right) \rightarrow \theta_{Z_{j}} \rightarrow \vartheta_{Z_{j-1}} \rightarrow 0
$$

is an exact sequence. From the long exact homology sequence for (1.4), it follows by induction that

$$
\begin{gathered}
H^{0}\left(M, \vartheta_{Z_{k}}\right)=\mathbf{C}, \quad 1 \leqslant k \leqslant l, \\
\operatorname{dim} H^{1}\left(M, \Theta_{Z_{k}}\right)=\sum \operatorname{dim} H^{1}\left(M, \vartheta\left(-Z_{j-1}\right) / \vartheta\left(-Z_{j}\right)\right), \quad 1 \leqslant j \leqslant k .
\end{gathered}
$$

Since $M$ is two dimensional and not compact,

$$
H^{2}(M, \mathscr{F})=0
$$

for any coherent sheaf $\mathcal{F}$ on $M[25]$.

Definition 1.1. A cycle $E>0$ is minimally elliptic if $\chi(E)=0$ and $\chi(D)>0$ for all cycles $D$ such that $0<D<E$.

Wagreich [27] defined the singularity $p$ to be elliptic if $\chi(D) \geqslant 0$ for all 
cycles $D>0$ and $\chi(F)=0$ for some cycles $F>0$. He proved that this definition is independent of the resolution. It is easy to see that under the hypothesis, $\chi(Z)=0$. The converse is also true [19], [21]. Henceforth, we will adopt the following definition.

Definition 1.2. $p$ is said to be weakly elliptic if $\chi(Z)=0$.

THEOREM 1.3 (LAUfER). Let $\pi: M \rightarrow V$ be the minimal resolution of the normal two-dimensional variety $V$ with one singular point $p$. Let $Z$ be the fundamental cycle on the exceptional set $A=\pi^{-1}(p)$. Then the following are equivalent:

(1) $Z$ is a minimally elliptic cycle.

(2) $A_{i} \cdot Z=-A_{i} \cdot K$ for all irreducible components $A_{i}$ in $A$.

(3) $\chi(Z)=0$ and any connected proper subvariety of $A$ is the exceptional set for a normal two-dimensional singularity.

Definition 1.4. Let $p$ be a normal two-dimensional singularity. $p$ is minimally elliptic if the minimal resolution $\pi: M \rightarrow V$ of a neighborhood of $p$ satisfies the conditions of Theorem 1.3.

THEOREM 1.5 (LAUfER). Let $\pi: M \rightarrow S$ represent $A$ as an exceptional set in the 2-dimensional manifold $M$ with $S$ a Stein space. Let $A=\cup_{i=1}^{n} A_{i}$ be the decomposition of $A$ into irreducible components and suppose that the $A_{i}$ are nonsingular. Let $\kappa_{i}$ be the canonical bundle of $A_{i}$ and $N_{i}$ the normal bundle. If $V$ is a line bundle over $M$ such that $C_{i}(V) \geqslant C\left(\kappa_{i} N_{i}^{*}\right)$, then $H^{1}(M, \mathcal{V})=0$ where $\mathcal{V}$ denotes the sheaf of germs of sections of the line bundle $V$.

Definition 1.6. Let $A$ be the exceptional set of the minimal good resolution $\pi: M \rightarrow V$ where $V$ is a normal two-dimensional Stein space with $p$ as its only weakly elliptic singularity. If $E \cdot Z<0$, we say that the elliptic sequence is $\{Z\}$ and the length of elliptic sequence is equal to one. Suppose $E \cdot Z=0$. Let $B_{1}$ be the maximal connected subvariety of $A$ such that $B_{1} \supseteq \operatorname{supp} E$ and $A_{i} \cdot Z=0, \forall A_{i} \subseteq B_{1}$. Since $A$ is an exceptional set, $Z \cdot Z<0$. So $B_{1}$ is properly contained in $A$. Suppose $Z_{B_{1}} \cdot E=0$. Let $B_{2}$ be the maximal connected subvariety of $B_{1}$ such that $B_{2} \supseteq|E|$ and $A_{i} \cdot Z_{B_{1}}=0 \forall A_{i} \subseteq B_{2}$. By the same argument as above, $B_{2}$ is properly contained in $B_{1}$. Continuing this process, we finally obtain $B_{m}$ with $Z_{B_{m}} \cdot E<0$. We call $\left\{Z_{B_{0}}=Z\right.$, $\left.Z_{B_{1}}, \ldots, Z_{B_{m}}\right\}$ the elliptic sequence and the length of elliptic sequence is $m+1$.

THEOREM 1.7. Let $\pi: M \rightarrow V$ be the minimal good resolution of normal two-dimensional Stein space with $p$ as its only weakly elliptic singularity. Suppose $p$ is not a minimally elliptic singularity and $K^{\prime}$ exists. Then the elliptic sequence is of the following form

$$
Z_{B_{0}}=Z, Z_{B_{1}}, \ldots, Z_{B_{l}}=Z_{E}, \quad l \geqslant 0 .
$$

Moreover, $-K^{\prime}=\sum_{i=0}^{l} Z_{B_{i}}+E$. 
2. Necessary and sufficient condition for the existence of Gorenstein structure for weakly elliptic singularities.

LEMMA 2.1. Let $\pi: M \rightarrow V$ be the minimal good resolution of normal two-dimensional Stein space with $p$ as its only weakly elliptic singular point. Suppose $K^{\prime}$ exists. Let $Z_{B_{0}}=Z, \ldots, Z_{B_{l}}, Z_{E}=Z_{B_{t+1}}$ be the elliptic sequence. Let $C_{j}=\sum_{i=0}^{j} Z_{B_{i}}$. Then either

$$
H^{0}\left(M, \vartheta\left(-C_{j}\right) / \mathcal{\vartheta}\left(-C_{j}-E\right)\right) \simeq 0 \simeq H^{1}\left(M, \vartheta\left(-C_{j}\right) / \vartheta\left(-C_{j}-E\right)\right)
$$

or

$$
H^{0}\left(M, \vartheta\left(-C_{j}\right) / \vartheta\left(-C_{j}-E\right)\right) \simeq \mathbf{C} \simeq H^{1}\left(M, \vartheta\left(-C_{j}\right) / \vartheta\left(-C_{j}-E\right)\right) .
$$

Proof. Choose a computation sequence for $Z$ as follows: $Z_{0}=0, Z_{1}=A_{i_{1}}$, $Z_{2}=Z_{1}+A_{i_{2}}, \ldots, Z_{k}=E=Z_{k-1}+A_{i_{k}}, \ldots$ Consider the following exact sequence.

$$
0 \rightarrow \theta_{C_{j}} \rightarrow \theta_{C_{j}+E} \rightarrow \vartheta\left(-C_{j}\right) / \theta\left(-C_{j}-E\right) \rightarrow 0 .
$$

We have the corresponding long cohomology exact sequence.

$$
\begin{aligned}
0 & \rightarrow H^{0}\left(M, \vartheta_{C_{j}}\right) \rightarrow H^{0}\left(M, \vartheta_{C_{j}+E}\right) \rightarrow H^{0}\left(M, \vartheta\left(-C_{j}\right) / \vartheta\left(-C_{j}-E\right)\right) \\
& \rightarrow H^{1}\left(M, \vartheta_{C_{j}}\right) \rightarrow H^{1}\left(M, \vartheta_{C_{j}+E}\right) \rightarrow H^{1}\left(M, \vartheta\left(-C_{j}\right) / \vartheta\left(-C_{j}-E\right)\right) \rightarrow 0 .
\end{aligned}
$$

Hence

$$
\begin{aligned}
\operatorname{dim} H^{0} & \left(M, \vartheta_{C_{j}}\right)-\operatorname{dim} H^{1}\left(M, \vartheta_{C_{j}}\right) \\
& -\operatorname{dim} H^{0}\left(M, \vartheta_{C_{j}+E}\right)+\operatorname{dim} H^{1}\left(M, \vartheta_{C_{j}+E}\right) \\
= & \operatorname{dim} H^{1}\left(M, \vartheta\left(-C_{j}\right) / \vartheta\left(-C_{j}-E\right)\right) \\
& -\operatorname{dim} H^{0}\left(M, \vartheta\left(-C_{j}\right) / \vartheta\left(-C_{j}-E\right)\right)
\end{aligned}
$$

i.e.

$$
\begin{aligned}
\chi\left(C_{j}\right)-\chi\left(C_{j}+E\right)= & \operatorname{dim} H^{1}\left(M, \vartheta\left(-C_{j}\right) / \vartheta\left(-C_{j}-E\right)\right) \\
& -\operatorname{dim} H^{0}\left(M, \vartheta\left(-C_{j}\right) / \vartheta\left(-C_{j}-E\right)\right) .
\end{aligned}
$$

However $\chi\left(C_{j}\right)-\chi\left(C_{j}+E\right)=E \cdot C_{j}=0$, therefore $\operatorname{dim} H^{1}\left(M, \vartheta\left(-C_{j}\right) / \vartheta\left(-C_{j}-E\right)\right)=\operatorname{dim} H^{0}\left(M, \vartheta\left(-C_{j}\right) / \vartheta\left(-C_{j}-E\right)\right)$.

Case (i). Support of $E=|E|$ consists of only one irreducible component. In this case, $E=A_{i_{1}}$ is an elliptic curve. Since $\theta\left(-C_{j}\right) / \theta\left(-C_{j}-E\right)$ is the sheaf of germs of sections of a line bundle of degree zero over an elliptic curve, by the Riemann-Roch Theorem, the assertion holds.

Case (ii). Support of $E$ has at least two irreducible components. Then all the irreducible components of the exceptional set are rational curves and $Z_{j-1} \cdot A_{i_{j}}=1$ for all $j \neq 1, k ; Z_{k-1} \cdot A_{i_{k}}=2$. Consider the following sheaf 
exact sequences:

$$
\begin{aligned}
& 0 \rightarrow \vartheta\left(-C_{j}-Z_{1}\right) / \vartheta\left(-C_{j}-E\right) \rightarrow \vartheta\left(-C_{j}\right) / \vartheta\left(-C_{j}-E\right) \\
& \rightarrow \theta\left(-C_{j}\right) / \theta\left(-C_{j}-Z_{1}\right) \rightarrow 0, \\
& 0 \rightarrow \vartheta\left(-C_{j}-Z_{2}\right) / \vartheta\left(-C_{j}-E\right) \rightarrow \vartheta\left(C_{j}-Z_{1}\right) / \vartheta\left(-C_{j}-E\right) \\
& \rightarrow \theta\left(-C_{j}-Z_{1}\right) / \theta\left(-C_{j}-Z_{2}\right) \rightarrow 0 \text {, } \\
& 0 \rightarrow \Theta\left(-C_{j}-Z_{k-1}\right) / \Theta\left(-C_{j}-E\right) \rightarrow \Theta\left(-C_{j}-Z_{k-2}\right) / \vartheta\left(-C_{j}-E\right) \\
& \rightarrow \mathcal{O}\left(-C_{j}-Z_{k-2}\right) / \mathcal{O}\left(-C_{j}-Z_{k-1}\right) \rightarrow 0 \quad(k-1) .
\end{aligned}
$$

Look at the corresponding long cohomology exact sequences. The long cohomology exact sequence corresponding to equation (1) gives

$$
\begin{aligned}
0 & \rightarrow H^{0}\left(M, \vartheta\left(-C_{j}-Z_{1}\right) / \vartheta\left(-C_{j}-E\right)\right) \rightarrow H^{0}\left(M, \vartheta\left(-C_{j}\right) / \vartheta\left(-C_{j}-E\right)\right) \\
& \rightarrow H^{0}\left(M, \vartheta\left(-C_{j}\right) / \vartheta\left(-C_{j}-Z_{1}\right)\right) \rightarrow H^{1}\left(M, \vartheta\left(-C_{j}-Z_{1}\right) / \vartheta\left(-C_{j}-E\right)\right) \\
& \rightarrow H^{1}\left(M, \vartheta\left(-C_{j}\right) / \vartheta\left(-C_{j}\right) / \vartheta\left(-C_{j}-E\right)\right) \\
& \rightarrow H^{1}\left(M, \vartheta\left(-C_{j}\right) / \vartheta\left(-C_{j}-Z_{1}\right)\right) \rightarrow 0 \ldots
\end{aligned}
$$

Since $\theta\left(-C_{j}\right) / \theta\left(-C_{j}-Z_{1}\right)$ is the sheaf of germs of sections of a line bundle of degree zero over a rational curve, we have $H^{0}\left(M, \theta\left(-C_{j}\right) / \theta\left(-C_{j}\right.\right.$ $\left.\left.-Z_{1}\right)\right) \simeq \mathbf{C}$ and $H^{1}\left(M, \vartheta\left(-C_{j}\right) / \theta\left(-C_{j}-Z_{1}\right)\right) \simeq 0$ by Serre duality and the Riemann-Roch Theorem. We claim that $H^{1}\left(M, \theta\left(-C_{j}-Z_{1}\right) / \theta\left(-C_{j}-\right.\right.$ $E)) \simeq \mathrm{C}$ and $H^{0}\left(M, \vartheta\left(-C_{j}-Z_{1}\right) / \vartheta\left(-C_{j}-E\right)\right) \simeq 0$. The long cohomology exact sequence corresponding to (2) gives

$$
\begin{aligned}
0 & \rightarrow H^{0}\left(M, \vartheta\left(-C_{j}-Z_{2}\right) / \vartheta\left(-C_{j}-E\right)\right) \\
& \stackrel{\varphi_{2}}{\rightarrow} H^{0}\left(M, \vartheta\left(-C_{j}-Z_{1}\right) / \vartheta\left(-C_{j}-E\right)\right) \\
& \rightarrow H^{0}\left(M, \vartheta\left(-C_{j}-Z_{1}\right) / \vartheta\left(-C_{j}-Z_{2}\right)\right) \\
& \rightarrow H^{1}\left(M, \vartheta\left(-C_{j}-Z_{2}\right) / \vartheta\left(-C_{j}-E\right)\right) \\
& \stackrel{\psi_{2}}{\rightarrow} H^{1}\left(M, \vartheta\left(-C_{j}-Z_{1}\right) / \vartheta\left(-C_{j}-E\right)\right) \\
& \rightarrow H^{1}\left(M, \vartheta\left(-C_{j}-Z_{1}\right) / \vartheta\left(-C_{j}-Z_{2}\right)\right) \rightarrow 0 .
\end{aligned}
$$

Since $\theta\left(-C_{j}-Z_{1}\right) / \theta\left(-C_{j}-Z_{2}\right)$ is the sheaf of germs of sections of a line bundle of degree -1 over a rational curve, we have

$$
\begin{aligned}
H^{0}\left(M, \vartheta\left(-C_{j}\right.\right. & \left.\left.-Z_{1}\right) / \vartheta\left(-C_{j}-Z_{2}\right)\right) \\
& \simeq 0 \simeq H^{1}\left(M, \vartheta\left(-C_{j}-Z_{1}\right) / \vartheta\left(-C_{j}-Z_{2}\right)\right) .
\end{aligned}
$$

So $\varphi_{2}$ and $\psi_{2}$ are isomorphisms. By exactly the same argument as above, one has the following isomorphisms. 


$$
\begin{aligned}
& H^{0}\left(M, \mathcal{O}\left(-C_{j}-Z_{i}\right) / \mathcal{O}\left(-C_{j}-E\right)\right) \\
& \stackrel{\varphi_{i}}{\rightarrow} H^{0}\left(M, \mathcal{O}\left(-C_{j}-Z_{i-1}\right) / \mathcal{O}\left(-C_{j}-E\right)\right), \\
& H^{1}\left(M, \mathcal{O}\left(-C_{j}-Z_{i}\right) / \mathcal{O}\left(-C_{j}-E\right)\right) \\
& \stackrel{\psi_{i}}{\rightarrow} H^{1}\left(M, \mathcal{O}\left(-C_{j}-Z_{i-1}\right) / \mathcal{O}\left(-C_{j}-E\right)\right)
\end{aligned}
$$

for $3<i<k-2$. The long cohomology sequence corresponding to $(k-1)$ gives

$$
\begin{aligned}
0 & \rightarrow H^{0}\left(M, \mathcal{O}\left(-C_{j}-Z_{k-1}\right) / \mathcal{O}\left(-C_{j}-E\right)\right) \\
& \rightarrow H^{0}\left(M, \mathcal{O}\left(-C_{j}-Z_{k-2}\right) / \mathcal{O}\left(-C_{j}-E\right)\right) \\
& \rightarrow H^{0}\left(M, \mathcal{O}\left(-C_{j}-Z_{k-2}\right) / \mathcal{O}\left(-C_{j}-Z_{k-1}\right)\right) \\
& \rightarrow H^{1}\left(M, \mathcal{O}\left(-C_{j}-Z_{k-1}\right) / \mathcal{O}\left(-C_{j}-E\right)\right) \\
& \rightarrow H^{1}\left(M, \mathcal{O}\left(-C_{j}-Z_{k-2}\right) / \mathcal{O}\left(-C_{j}-E\right)\right) \\
& \rightarrow H^{1}\left(M, \mathcal{O}\left(-C_{j}-Z_{k-2}\right) / \mathcal{O}\left(-C_{j}-Z_{k-1}\right)\right) \rightarrow 0 .
\end{aligned}
$$

Since $\theta\left(-C_{j}-Z_{k-1}\right) / \mathcal{O}\left(-C_{j}-E\right)$ and $\theta\left(-C_{j}-Z_{k-2}\right) / \mathcal{O}\left(-C_{j}-Z_{k-1}\right)$ are the sheaf of germs of sections of line bundles of degree -2 and -1 respectively over rational curves, we have

$$
\begin{aligned}
& H^{0}\left(M, \mathcal{O}\left(-C_{j}-Z_{k-1}\right) / \mathcal{O}\left(-C_{j}-E\right)\right) \simeq 0 \\
& H^{1}\left(M, \mathcal{O}\left(-C_{j}-Z_{k-1}\right) / \mathcal{O}\left(-C_{j}-E\right)\right) \simeq \mathbf{C} \\
& H^{0}\left(M, \mathcal{O}\left(-C_{j}-Z_{k-2}\right) / \mathcal{O}\left(-C_{j}-Z_{k-1}\right)\right) \simeq 0 \\
& \quad \simeq H^{1}\left(M, \mathcal{O}\left(-C_{j}-Z_{k-2}\right) / \mathcal{O}\left(-C_{j}-Z_{k-1}\right)\right) .
\end{aligned}
$$

Hence

$$
\begin{gathered}
H^{0}\left(M, \mathcal{O}\left(-C_{j}-Z_{k-2}\right) / \mathcal{O}\left(-C_{j}-E\right)\right) \simeq 0 \text { and } \\
H^{1}\left(M, \mathcal{O}\left(-C_{j}-Z_{k-2}\right) / \mathcal{O}\left(-C_{j}-E\right)\right) \simeq \mathbf{C} .
\end{gathered}
$$

This proves our claim. From (*) we have the exact sequence

$$
\begin{aligned}
0 & \rightarrow H^{0}\left(M, \vartheta\left(-C_{j}\right) / \mathcal{O}\left(-C_{j}-E\right)\right) \\
& \rightarrow H^{0}\left(M, \mathcal{O}\left(-C_{j}\right) / \mathcal{O}\left(-C_{j}-Z_{1}\right)\right) \simeq \mathbf{C} \\
& \rightarrow H^{1}\left(M, \mathcal{O}\left(-C_{j}-Z_{1}\right) / \mathcal{O}\left(-C_{j}-E\right)\right) \simeq \mathbf{C} \\
& \rightarrow H^{1}\left(M, \mathcal{O}\left(-C_{j}\right) / \mathcal{O}\left(-C_{j}-E\right)\right) \rightarrow 0 .
\end{aligned}
$$

The lemma follows easily from the above exact sequence. Q.E.D. 
Let $\pi: M \rightarrow V$ be a resolution of a normal two-dimensional Stein space $V$ with $p$ as its only singularity. Serre duality gives $H^{1}(M, \theta)$ as dual to $H_{c}^{1}(M, \Omega)$, where $\Omega$ is the canonical sheaf, i.e. the sheaf of germs of holomorphic 2-forms. By [16, Theorem 3.4, p. 604], for suitable $M$, which can be chosen to be arbitrarily small neighborhoods of $A=\pi^{-1}(p), H_{c}^{1}(M, \Omega)$ may be identified with $H^{0}(M \backslash A, \Omega) / H^{0}(M, \Omega)$. Let $\omega \in H^{0}(M \backslash A, \Omega)$. $H_{c}^{1}(M, \Omega)$ is a $H^{0}(M, \vartheta)$-module of finite dimension over C. So $\omega$ is meromorphic on $M$ with possible poles on the $A_{i}$. The corresponding element in $H_{c}^{1}(M, \Omega)$ is given by restricting $\omega$ to $M \backslash N_{0}$, where $N_{0} \subset \subset M$ is a neighborhood of $A$, extending $\omega$ to a $C^{\infty}(2,0)$-form $\tilde{\omega}$ on $M$ and then taking $\operatorname{cls}[\bar{\partial} \tilde{\omega}] \in H_{c}^{1}(M, \Omega)$. If $\tilde{\lambda}$ is a $\bar{\partial}$-closed $C^{\infty}(0,1)$-form, the pairing between $\operatorname{cls}[\tilde{\lambda}] \in H^{1}(M, \Theta)$ and $\operatorname{cls}[\bar{\partial} \tilde{\omega}]$ is given by

$$
\int_{M} \tilde{\lambda} \wedge \bar{\partial} \tilde{\omega}=\langle\operatorname{cls}[\tilde{\lambda}], \operatorname{cls}[\bar{\partial} \tilde{\omega}]\rangle
$$

The duality of the pairing in (3.4) holds for all holomorphically convex $M^{\prime} \supset N_{0}$ since the restriction map is an isomorphism on $H^{1}(M, \theta)$ [16, Lemma 3.1, p. 599].

LeMma 2.2 (LAUfER). Let $A_{i}, 1 \leqslant i \leqslant n$, be the irreducible component of $A$. Assume that the $A_{i}$ are nonsingular with normal crossings. Let $A^{\prime}=\cup A_{i}$, $2 \leqslant i \leqslant n$. Let $P_{1}, \ldots, P_{t}$ be disjoint polydisc coordinate patches on $M$ such that

(i) $A_{1} \cap A^{\prime} \subset \cup P_{j}, 1 \leqslant j \leqslant t$,

(ii) in the $\left(x_{j}, y_{j}\right)$ coordinate system on $P_{j}, A_{1}=\left\{y_{j}=0\right\}$,

(iii) if $A^{\prime} \cap P_{j} \neq \varnothing$, then $A \cap P_{j}=\left\{x_{j} y_{j}=0\right\}$.

Let $N, N_{0} \subset \subset N \subset \subset M$, have a cover $\mathcal{U}=\left\{U_{0}, U_{1}\right\}$ where $U_{0}$ contains $\cup P_{j}, 1 \leqslant j \leqslant t$, and is also a neighborhood of $A^{\prime} . U_{1} \cap A_{1}$ should be the complement of discs in the $x_{j}$-coordinate systems of (ii). Suppose that $U_{0} \cap U_{1}$ $=\bigcup U_{0_{j}}, 1 \leqslant j \leqslant t$, with $U_{0_{j}}=\left\{\left(x_{j}, y_{j}\right) \in P_{j}: r<\left|x_{j}\right|<R,\left|y_{j}\right|<R\right\}$ for suitable $r$ and $R$. Let $\mathcal{U}^{\prime}=\left\{U_{0}^{\prime}, U_{1}^{\prime}\right\}$ have $U_{k} \subset \subset U_{k}^{\prime}, k=0,1$. Let $\lambda=\left\{\lambda_{0_{j}}\right.$ $\left.\in H^{0}\left(U_{0_{j}}^{\prime}, \vartheta\right)\right\}$ be a cocycle in $H^{1}\left(N\left(\mathcal{U}^{\prime}\right), \vartheta\right)$. Upon restricting to $M^{\prime}, N_{0} \subset \subset$ $M^{\prime} \subset \subset N, \lambda$ determines an element $\operatorname{cls}[\lambda]$ in $H^{1}\left(M^{\prime}, \theta\right)$. Then the pairing in (1.1) between $\operatorname{cls}[\lambda]$ and $\operatorname{cls}[\bar{\partial} \tilde{\omega}]$ over $M^{\prime}$ is given by

$$
\langle\operatorname{cls}[\lambda], \operatorname{cls}[\omega]\rangle=\sum_{j=1}^{t} \int_{\substack{\left|x_{j}\right|=R \\\left|y_{j}\right|=R}} \lambda_{0_{j}} \omega .
$$

Proof. $\tilde{\lambda}$ is obtained by finding $C^{\infty}$ functions $\lambda_{k}$ on $U_{k}^{\prime}, k=0,1$, such that $\lambda_{0_{j}}=\lambda_{1}-\lambda_{0}$ on $U_{0_{j}}^{\prime} \cdot \tilde{\lambda}=\bar{\partial} \lambda_{k}$ on $U_{k}^{\prime} \cdot \bar{\partial} \tilde{\omega}=0$ outside of $N_{0}$, so 


$$
\begin{aligned}
\langle\lambda, \omega\rangle & =\int_{M^{\prime}} \tilde{\lambda} \wedge \bar{\partial} \tilde{\omega}=\int_{N} \tilde{\lambda} \wedge \bar{\partial} \tilde{\omega}=-\int_{\partial N} \tilde{\lambda} \wedge \tilde{\omega} \\
& =-\int_{\partial N} \tilde{\lambda} \wedge \omega=-\sum_{k=0}^{1} \int_{\partial N \cap \bar{U}_{k}} \bar{\partial} \lambda_{k} \wedge \omega+\sum_{j=1}^{t} \int_{\partial N \cap \bar{O}_{0, j}} \bar{\partial} \lambda_{1} \wedge \omega \\
& =-\sum_{k=0}^{1} \int_{\partial\left(\partial N \cap U_{k}\right)} \lambda_{k} \wedge \omega+\sum_{j=1}^{t} \int_{\partial\left(\partial N \cap U_{0_{j}}\right)} \lambda_{1} \wedge \omega \\
& =\sum_{j=1}^{t} \int_{\substack{\left|x_{j}\right|=R \\
\left|y_{j}\right|=R}}\left(\lambda_{1}-\lambda_{0}\right) \wedge \omega=\sum_{j=1}^{t} \int_{\substack{\left|x_{j}\right|=R \\
\left|y_{j}\right|=R}} \lambda_{0_{j}} \cdot \omega . \quad \text { Q.E.D. }
\end{aligned}
$$

THEOREM 2.3. Let $\pi: M \rightarrow V$ be the minimal good resolution of normal two-dimensional Stein space with $p$ as its only weakly elliptic singular point. Suppose $K^{\prime}$ exists. Let $Z_{B_{0}}=Z, \ldots, Z_{B_{l+1}}$ be the elliptic sequence. Let $C_{l}=$ $\sum_{i=0}^{l} Z_{B_{i}}$. Then ${ }_{v} \mathcal{\theta}_{p}$ is Gorenstein if and only if $\varphi: H^{0}\left(M, \theta_{C_{i}+E}\right) \rightarrow$ $H^{0}\left(M, \Theta_{C_{l}}\right)$ is surjective and $\mathcal{O}\left(-C_{l}\right) / \mathcal{O}\left(-C_{l}-E\right)$ is the sheaf of germs of sections of a trivial line bundle over $\left(|E|, \Theta_{E}\right)$.

Proof. " $\Rightarrow "$. Choose a computation sequence for $Z$ as follows: $Z_{0}=0$, $Z, \ldots, Z_{k}=E, \ldots$ By Theorem 3.7 of $[30]-K^{\prime}=C_{l}+E$. So $H^{1}\left(M, \vartheta\left(-C_{l}-E\right)\right)=0$. The exact sequence

$$
\begin{aligned}
H^{1}\left(M, \mathcal{O}\left(-C_{l}-E\right)\right) & \rightarrow H^{1}\left(M, \mathcal{\vartheta}\left(-C_{l}\right)\right) \\
& \stackrel{\psi}{\rightarrow} H^{1}\left(M, \vartheta\left(-C_{l}\right) / \mathcal{O}\left(-C_{l}-E\right)\right) \rightarrow 0
\end{aligned}
$$

shows that $\psi$ is an isomorphism. By Lemma 2.1 we have either

$$
H^{0}\left(M, \vartheta\left(-C_{l}\right) / \vartheta\left(-C_{l}-E\right)\right) \simeq 0 \simeq H^{1}\left(M, \vartheta\left(-C_{l}\right) / \vartheta\left(-C_{l}-E\right)\right)
$$

or

$$
H^{0}\left(M, \vartheta\left(-C_{l}\right) / \vartheta\left(-C_{l}-E\right)\right) \simeq \mathbf{C} \simeq H^{1}\left(M, \vartheta\left(-C_{l}\right) / \vartheta\left(-C_{l}-E\right)\right) .
$$

Thus either $H^{1}\left(M, \theta\left(-C_{l}\right)\right) \simeq \mathrm{C}$ or $H^{1}\left(M, \theta\left(-C_{l}\right)\right)=0$. We claim that $H^{1}\left(M, \vartheta\left(-C_{l}\right)\right) \simeq \mathbf{C}$ and $H^{1}\left(M, \vartheta\left(-C_{l}\right)\right) \rightarrow H^{1}(M, \theta)$ is injective. Otherwise $H^{1}\left(M, \theta\left(-C_{l}\right)\right) \rightarrow H^{1}(M, \theta)$ is a zero map. As $v_{p}$ is Gorenstein, there exist $\omega \in H^{1}(M-A, \Omega)$ having no zeros near $A$ and the image of $\omega$ in $H^{0}(M-A, \Omega) / H^{0}(M, \Omega)$ is nonzero. Let $w_{i}$ be the order of the pole of $\omega$ on $A_{i}$. Consider a cover as in Lemma 1.2. On $P_{1}$ where $A_{1} \subseteq|E|$

$$
\omega=\frac{\omega_{1}\left(x_{1}, y_{1}\right)}{y_{1}^{w_{1}}} d x_{1} \wedge d y_{1}
$$

where $\omega_{1}\left(x_{1}, y_{1}\right)$ is a holomorphic function, $\omega_{1}\left(x_{1}, 0\right) \neq 0$. There is a holomorphic function $f\left(x_{1}\right) r \leqslant\left|x_{1}\right| \leqslant R$ such that 


$$
\int_{\substack{\left|x_{1}\right|=R \\\left|y_{1}\right|=R}} y_{1}^{w_{1}-1} f\left(x_{1}\right) \frac{\omega_{1}\left(x_{1}, y_{1}\right)}{y_{1}^{w_{1}}} d x_{1} \wedge d y_{1} \neq 0 .
$$

Let $\lambda_{0_{1}}=y_{1}^{w_{1}-1} f\left(x_{1}\right)$ and $\lambda_{0_{j}}=0$ for $2 \leqslant j \leqslant t$. Then by Lemma $2.2, \operatorname{cls}[\lambda] \neq$ 0 in $H^{1}\left(M^{\prime}, \theta\right)$. Let $E=\sum e_{i} A_{i}, Z_{B_{i}}=\sum_{j i} z_{j} A_{j}$. Then $w_{1}=\sum_{i=0}^{l} z_{1}+e_{1}$ and $w_{1}-1 \geqslant \sum_{i=0}^{l} z_{1}$. Hence $\lambda$ may be thought of as also a cocycle in $H^{1}\left(N(\mathcal{Q}), \theta\left(-C_{l}\right)\right)$. It follows that $\operatorname{cls}[\lambda]=0$ in $H^{1}\left(M^{\prime}, \theta\right)$. This leads to a contradiction. Our claim is proved. Consider the following commutative diagram with exact rows.

$$
\begin{aligned}
& 0 \rightarrow H^{0}\left(M, \theta\left(-C_{l}-E\right)\right) \rightarrow H^{0}(M, \theta) \rightarrow H^{0}\left(M, \theta_{C_{l}+E}\right) \\
& \downarrow \quad \downarrow \quad \downarrow \\
& 0 \rightarrow \quad H^{0}\left(M, \mathcal{O}\left(-C_{l}\right)\right) \rightarrow H^{0}(M, \mathcal{O}) \rightarrow H^{0}\left(M, \vartheta_{C_{l}}\right) \\
& \rightarrow H^{1}\left(M, \vartheta\left(-C_{l}-E\right)\right) \rightarrow H^{1}(M, \vartheta) \rightarrow H^{1}\left(M, \theta_{C_{l}+E}\right) \rightarrow 0 \\
& \downarrow \quad \downarrow \quad \downarrow \\
& \rightarrow H^{1}\left(M, \vartheta\left(-C_{l}\right)\right) \rightarrow H^{1}(M, \vartheta) \rightarrow H^{1}\left(M, \theta_{C_{l}}\right) \rightarrow 0
\end{aligned}
$$

It follows that $H^{0}\left(M, \theta_{C_{l}}\right) \rightarrow H^{0}\left(M, \theta_{C_{l}}\right)$ is surjective. Look at the following exact sequence

$$
\begin{aligned}
0 \rightarrow H^{0}\left(M, \vartheta\left(-C_{l}-E\right)\right) & \rightarrow H^{\mathrm{v}}\left(M, \vartheta\left(-C_{l}\right)\right) \\
& \rightarrow H^{0}\left(M, \vartheta\left(-C_{l}\right) / \vartheta\left(-C_{l}-E\right)\right) \simeq \mathbf{C} \rightarrow 0 .
\end{aligned}
$$

Let $\theta\left(-C_{l}\right) / \theta\left(-C_{l}-E\right)$ correspond to a line bundle $L$ over $\left(|E|, \theta_{E}\right)$. There exists $f \in H^{0}\left(M, \theta\left(-C_{l}\right)\right)$ such that the image of $f$ in $H^{0}\left(M, \theta\left(-C_{l}\right) / \theta\left(-C_{l}-E\right)\right)$ viewed as a section of line bundle $L$ is nowhere zero. So $L$ is a trivial bundle.

" $\Leftarrow$ " Suppose conversely that $H^{0}\left(M, \theta_{C_{1}+E}\right) \rightarrow H^{0}\left(M, \theta_{C_{l}}\right)$ is surjective and $\theta\left(-C_{l}\right) / \theta\left(-C_{l}-E\right)$ is the sheaf of germs of sections of a trivial line bundle over $\left(|E|, \theta_{E}\right)$. Then

$$
H^{0}\left(M, \vartheta\left(-C_{l}\right) / \theta\left(-C_{l}-E\right)\right) \simeq \mathbf{C} \simeq H^{1}\left(M, \theta\left(-C_{l}\right) / \theta\left(-C_{l}-E\right)\right) .
$$

The exact sequence

$$
\begin{aligned}
0 \simeq H^{1}\left(M, \vartheta\left(-C_{l}-E\right)\right) & \rightarrow H^{1}\left(M, \vartheta\left(-C_{l}\right)\right) \\
& \rightarrow H^{1}\left(M, \vartheta\left(-C_{l}\right) / \vartheta\left(-C_{l}-E\right)\right) \rightarrow 0
\end{aligned}
$$

shows that $H^{1}\left(M, \theta\left(-C_{l}\right)\right) \rightarrow H^{1}\left(M, \vartheta\left(-C_{l}\right) / \theta\left(-C_{l}-E\right)\right) \simeq \mathbf{C}$ is an isomorphism. Consider the following commutative diagram with exact rows. 


$$
\begin{aligned}
& 0 \rightarrow H^{0}\left(M, \vartheta\left(-C_{l}-E\right)\right) \rightarrow H^{0}(M, \vartheta) \rightarrow H^{0}\left(M, \theta_{C_{l}+E}\right) \\
& \downarrow \quad \downarrow \quad \downarrow \\
& 0 \rightarrow \quad H^{0}\left(M, \vartheta\left(-C_{l}\right)\right) \rightarrow H^{0}(M, \vartheta) \rightarrow H^{0}\left(M, \theta_{C_{l}}\right) \\
& \rightarrow H^{1}\left(M, \vartheta\left(-C_{l}-E\right)\right) \rightarrow H^{1}(M, \vartheta) \rightarrow H^{1}\left(M, \Theta_{C_{l}+E}\right) \rightarrow 0 \\
& \downarrow \\
& \rightarrow \quad H^{1}\left(M, \vartheta\left(-C_{l}\right)\right) \rightarrow H^{1}(M, \vartheta) \rightarrow H^{1}\left(M, \theta_{C_{l}}\right)
\end{aligned}
$$

Since $H^{0}\left(M, \theta_{C_{l}+E}\right) \rightarrow H^{0}\left(M, \theta_{C_{l}}\right)$ is surjective, $H^{0}(M, \theta) \rightarrow H^{0}\left(M, \theta_{C_{l}}\right)$ is also surjective. So $H^{1}\left(M, \theta\left(-C_{l}\right)\right) \simeq \mathbf{C} \rightarrow H^{1}(M, \theta)$ is injective. Since $H^{1}\left(M, \Theta\left(-C_{l}\right) / \mathcal{O}\left(-C_{l}-E\right)\right) \simeq \mathbf{C}$, the usual long cohomology exact sequence argument shows that

$$
\begin{aligned}
H^{1}\left(M, \vartheta\left(-C_{l}-Z_{k-1}\right) / \vartheta\left(-C_{l}-E\right)\right) \simeq \mathbf{C} \\
\rightarrow H^{1}\left(M, \vartheta\left(-C_{l}\right) / \vartheta\left(-C_{l}-E\right)\right)
\end{aligned}
$$

is an isomorphism. Look at the following commutative diagram with exact rows.

$$
\begin{aligned}
& H^{1}\left(M, \vartheta\left(-C_{l}-Z_{k-1}\right)\right) \stackrel{\alpha}{\rightarrow} H^{1}\left(M, \vartheta\left(-C_{l}-Z_{k-1}\right) / \mathcal{\Theta}\left(-C_{l}-E\right)\right) \simeq \mathbf{C} \rightarrow 0 \\
& \downarrow \boldsymbol{\beta} \\
& H^{1}\left(M, \vartheta\left(-C_{l}\right)\right) \simeq \mathbf{C} \rightarrow \quad H^{1}\left(M, \vartheta\left(-C_{l}\right) / \vartheta\left(-C_{l}-E\right)\right) \simeq \mathbf{C} \quad \rightarrow 0 \\
& \downarrow \gamma \\
& H^{1}(M, \theta)
\end{aligned}
$$

There exists $\lambda \in H^{1}\left(M, \mathcal{O}\left(-C_{l}-Z_{k-1}\right)\right)$ such that $\alpha(\lambda) \neq 0 \neq \gamma \cdot \beta(\lambda)$. Use the notation of Lemma 2.2. $Q$ is a Leray cover for $A_{1}$. So there exists $\left\{\lambda_{0 i}=y_{1}^{\Sigma_{j-0}^{\prime} z_{1}+e_{1}-1} \cdot f_{i}\left(x_{1}\right)+\right.$ higher power of $\left.y_{1}\right\}, f_{i}\left(x_{1}\right)$ is holomorphic for $r<\left|x_{1}\right|<R$ such that $\operatorname{cls}[\lambda] \neq 0$ in $H^{1}\left(M^{\prime}, \theta\right)$. Let $\omega$ be the element such that $\langle\lambda, \omega\rangle \neq 0$. Then on $P_{1}$ by Lemma 2.2, we know that $w_{1}>\sum_{i=0}^{l} z_{1}+e_{1}$ - 1 where $w_{1}$ is the order of pole of $\omega$ on $A_{1} .(\omega)=[\omega]+D$ where $D$ is a positive divisor which does not involve any $A_{i}$ and $[\omega]=-\Sigma_{i} w_{i} A_{i}$. For any

$$
\begin{aligned}
A_{i} & \subseteq A,-A_{i} \cdot\left(\sum_{i=0} Z_{B_{i}}+E\right)=A_{i} \cdot(\omega)=A_{i} \cdot[\omega]+A_{i} \cdot D, \\
& \Rightarrow A_{i} \cdot\left([\omega]+\sum_{i=0} Z_{B_{i}}+E+D\right)=0 \text { for all } A_{i} \subseteq A \\
& \Rightarrow A_{i} \cdot\left(\sum_{i=0} Z_{B_{i}}+E+[\omega]\right) \leqslant 0 \text { for all } A_{i} \subseteq A
\end{aligned}
$$

Let $Y=\sum_{i=0}^{l} Z_{B_{i}}+E+[\omega]=\sum y_{i} A_{i}$. We have $y_{1} \leqslant 0$ and $A_{i} \cdot Y \leqslant 0$ for all $A_{i} \subseteq A$. By the proof of Theorem 3.11 of [30] this is possible only if $Y=0$. It follows easily that $D=0$ and $(\omega)=-\sum_{i=0}^{l} Z_{B_{i}}-E$. So $\omega$ has no zeros in an $n$ neighborhood of $A$, i.e., $V_{P} \vartheta_{P}$ is Gorenstein. Q.E.D. 
THEOREM 2.4. Let $\pi: M \rightarrow V$ be the minimal good resolution of normal twodimensional Stein space $V$ with $p$ as its only weakly elliptic singularity. Suppose $K^{\prime}$ exists. Let $Z_{B_{0}}=Z, \ldots, Z_{B_{l}}, Z_{E}$ be the elliptic sequence. Then $p$ is a maximally elliptic singularity if and only if $H^{0}\left(M, \theta_{C_{j}+E}\right) \rightarrow H^{0}\left(M, \theta_{C_{j}}\right)$ is surjective for $0 \leqslant j \leqslant l$ and $\theta\left(-C_{j}\right) / \theta\left(-C_{j}-E\right)$ is the sheaf of germs of sections of a trivial line bundle over $\left(|E|, \theta_{E}\right)$ for $0 \leqslant j \leqslant l$.

Proof. Let us first prove that $\operatorname{dim} H^{0}\left(M, \theta_{C_{j}+E}\right)-1 \leqslant \operatorname{dim} H^{0}\left(M, \theta_{C_{j}}\right)$ $\leqslant \operatorname{dim} H^{0}\left(M, \theta_{C_{j}+E}\right)$. We recall that $\chi\left(C_{j}+E\right)=0=\chi\left(C_{j}\right)$. The exact sequence

$$
H^{1}\left(M, \Theta_{C_{j}+E}\right) \rightarrow H^{1}\left(M, \theta_{C_{j}}\right) \rightarrow 0
$$

shows that

$$
\begin{aligned}
\operatorname{dim} H^{0}\left(M, \theta_{C_{j}}\right) & =\operatorname{dim} H^{1}\left(M, \theta_{C_{j}}\right) \leqslant \operatorname{dim} H^{1}\left(M, \theta_{C_{j}+E}\right) \\
& =\operatorname{dim} H^{0}\left(M, \theta_{C_{j}+E}\right) .
\end{aligned}
$$

By the proof of the previous theorem, we know that either

$$
H^{0}\left(M, \vartheta\left(-C_{j}\right) / \vartheta\left(-C_{j}-E\right)\right) \simeq 0 \simeq H^{1}\left(M, \vartheta\left(-C_{j}\right) / \vartheta\left(-C_{j}-E\right)\right)
$$

or

$$
H^{0}\left(M, \vartheta\left(-C_{j}\right) / \vartheta\left(-C_{j}-E\right)\right) \simeq \mathbf{C} \simeq H^{1}\left(M, \vartheta\left(-C_{j}\right) / \vartheta\left(-C_{j}-E\right)\right) .
$$

The exact sequence

$$
\begin{aligned}
0 & \rightarrow H^{0}\left(M, \vartheta\left(-C_{j}\right) / \vartheta\left(-C_{j}-E\right)\right) \rightarrow H^{0}\left(M, \Theta_{C_{j}+E}\right) \rightarrow H^{0}\left(M, \Theta_{C_{j}}\right) \\
& \rightarrow H^{1}\left(M, \vartheta\left(-C_{j}\right) / \vartheta\left(-C_{j}-E\right)\right)
\end{aligned}
$$

shows that $\operatorname{dim} H^{0}\left(M, \theta_{C_{j}}\right) \geqslant \operatorname{dim} H^{1}\left(M, \theta_{C_{j}+E}\right)-1$. Choose a computation sequence for $Z_{B_{j+1}}$ as follows $Z_{0}=0, Z_{1}, \ldots, Z_{k}=E, \ldots, Z_{l}=Z_{B_{j+1}}$. Consider the following sheaf exact sequence.

$$
\begin{aligned}
& 0 \rightarrow \Theta\left(-C_{j}-Z_{k+1}\right) / \Theta\left(-C_{j+1}\right) \rightarrow \Theta\left(-C_{j}-E\right) / \Theta\left(-C_{j}-Z_{B_{j+1}}\right) \\
& \rightarrow \quad \vartheta\left(-C_{j}-E\right) / \mathcal{O}\left(-C_{j}-Z_{k+1}\right) \rightarrow 0 \text {, } \\
& 0 \rightarrow \theta\left(-C_{j}-Z_{k+2}\right) / \theta\left(-C_{j+1}\right) \rightarrow \theta\left(-C_{j}-Z_{k+1}\right) / \theta\left(-C_{j+1}\right) \\
& \rightarrow \theta\left(-C_{j}-Z_{k+1}\right) / \theta\left(-C_{j}-Z_{k+2}\right) \rightarrow 0 \text {, } \\
& 0 \rightarrow \mathcal{O}\left(-C_{j}-Z_{l-1}\right) / \mathcal{O}\left(-C_{j+1}\right) \rightarrow \mathcal{\Theta}\left(-C_{j}-Z_{l-2}\right) / \mathcal{O}\left(-C_{j+1}\right) \\
& \rightarrow \quad \theta\left(-C_{j}-Z_{l-2}\right) / \theta\left(-C_{j}-Z_{l-1}\right) \rightarrow 0 \text {. }
\end{aligned}
$$

By the usual long cohomology exact sequence argument, $H^{0}\left(M, \vartheta\left(-C_{j}-E\right) / \mathcal{O}\left(-C_{j+1}\right)\right) \simeq 0 \simeq H^{1}\left(M, \vartheta\left(-C_{j}-E\right) / \vartheta\left(-C_{j+1}\right)\right)$. 
The following two exact sequences

$$
\begin{aligned}
0 & \rightarrow \quad H^{0}\left(M, \vartheta\left(-C_{j}-E\right) / \vartheta\left(-C_{j+1}\right)\right) \rightarrow H^{0}\left(M, \Theta_{C_{j+1}}\right) \\
& \rightarrow \quad H^{0}\left(M, \vartheta_{C_{j}+E}\right) \rightarrow H^{1}\left(M, \vartheta\left(-C_{j}-E\right) / \vartheta\left(-C_{j+1}\right)\right) \\
& \rightarrow \quad H^{1}\left(M, \vartheta_{C_{j+1}}\right) \rightarrow H^{1}\left(M, \vartheta_{C_{j}+E}\right) \rightarrow 0, \\
0 \quad & \rightarrow \quad H^{0}\left(M, \vartheta\left(-C_{j+1}\right)\right) \rightarrow H^{0}\left(M, \vartheta\left(-C_{j}-E\right)\right) \\
& \rightarrow \quad H^{0}\left(M, \vartheta\left(-C_{j}-E\right) / \vartheta\left(-C_{j+1}\right)\right) \rightarrow H^{1}\left(M, \vartheta\left(-C_{j+1}\right)\right) \\
& \rightarrow \quad H^{1}\left(M, \vartheta\left(-C_{j}-E\right)\right) \rightarrow H^{1}\left(M, \vartheta\left(-C_{j}-E\right) / \vartheta\left(-C_{j+1}\right)\right) \rightarrow 0
\end{aligned}
$$

shows that $H^{d}\left(M, \Theta_{C_{j+1}}\right) \rightarrow H^{d}\left(M, \Theta_{C_{j+1}+E}\right)$ and $H^{d}\left(M, \theta\left(-C_{j+1}\right)\right) \rightarrow$ $H^{d}\left(M, \mathcal{O}\left(-C_{j}-E\right)\right)$ are isomorphisms for $d=0,1$.

Suppose $p$ is a maximally elliptic singularity. Consider the following commutative diagram with exact rows.

$$
\begin{aligned}
& 0 \rightarrow H^{0}\left(M, \vartheta\left(-C_{l}-E\right)\right) \rightarrow H^{0}(M, \vartheta) \rightarrow H^{0}\left(M, \theta_{C_{l}+E}\right) \\
& \downarrow \quad \downarrow \quad \downarrow \\
& 0 \rightarrow H^{0}\left(M, \mathcal{\theta}\left(-C_{l}\right)\right) \rightarrow H^{0}(M, \vartheta) \rightarrow H^{0}\left(M, \theta_{C_{l}}\right) \\
& 0 \rightarrow H^{0}\left(M, \vartheta\left(-C_{j+1}\right)\right) \rightarrow H^{0}(M, \vartheta) \rightarrow H^{0}\left(M, \theta_{C_{j+1}}\right)
\end{aligned}
$$

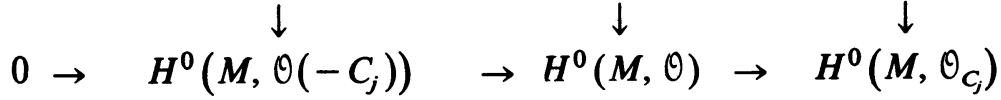

$$
\begin{aligned}
& 0 \rightarrow H^{0}(M, \vartheta(-Z)) \rightarrow H^{0}(M, \theta) \rightarrow H^{0}\left(M, \theta_{z}\right) \\
& \rightarrow H^{1}\left(M, \vartheta\left(-C_{l}-E\right)\right) \rightarrow H^{1}(M, \vartheta) \rightarrow H^{1}\left(M, \theta_{C_{l}+E}\right) \rightarrow 0 \\
& \downarrow \\
& \rightarrow H^{1}\left(M, \theta\left(-C_{l}\right)\right) \quad \rightarrow H^{1}(M, \vartheta) \rightarrow H^{1}\left(M, \theta_{C_{l}}\right) \rightarrow 0 \\
& \rightarrow H^{1}\left(M, \theta\left(-C_{j+1}\right)\right) \rightarrow H^{1}(M, \vartheta) \rightarrow H^{1}\left(M, \theta_{C_{j+1}}\right) \rightarrow 0 \\
& \downarrow \downarrow \downarrow \downarrow \downarrow \\
& \rightarrow H^{1}\left(M, \vartheta\left(-C_{j}\right)\right) \quad \rightarrow H^{1}(M, \vartheta) \rightarrow H^{1}\left(M, \theta_{C_{j}}\right) \rightarrow 0 \\
& \rightarrow H^{1}(M, \vartheta(-Z)) \quad \rightarrow H^{1}(M, \theta) \rightarrow H^{1}\left(M, \theta_{Z}\right) \quad \rightarrow 0
\end{aligned}
$$


Since $H^{1}\left(M, \vartheta\left(-C_{l}-E\right)\right)=0, \operatorname{dim} H^{0}\left(M, \theta_{C_{l}+E}\right)=\operatorname{dim} H^{1}\left(M, \theta_{C_{l}+E}\right)=$ $\operatorname{dim} H^{1}(M, \theta)=l+2$. It follows that $H^{0}\left(M, \theta_{C_{j+1}}\right) \rightarrow H^{0}\left(M, \theta_{C_{j}}\right)$ is surjective for all $0 \leqslant j \leqslant l$ and $\operatorname{dim} H^{0}\left(M, \theta_{C_{j+1}}\right)=\operatorname{dim} H^{0}\left(M, \theta_{C_{j}}\right)+1$. Moreover, $H^{0}\left(M, \vartheta\left(-C_{j+1}\right)\right) \rightarrow H^{0}\left(M, \vartheta\left(-C_{j}\right)\right)$ is not an isomorphism for all $0 \leqslant j \leqslant l$. The exact sequence

$$
\begin{aligned}
0 & \rightarrow H^{0}\left(M, \vartheta\left(-C_{j}\right) / \vartheta\left(-C_{j}-E\right)\right) \\
& \rightarrow H^{0}\left(M, \vartheta_{C_{j}+E}\right) \rightarrow H^{0}\left(M, \vartheta_{C_{j}}\right) \rightarrow 0
\end{aligned}
$$

shows that $H^{0}\left(M, \vartheta\left(-C_{j}\right) / \vartheta\left(-C_{j}-E\right)\right) \simeq \mathbf{C}$. We have the following exact sequence

$$
\begin{aligned}
0 \rightarrow H^{0}\left(M, \vartheta\left(-C_{j}-E\right)\right) & \rightarrow H^{0}\left(M, \vartheta\left(-C_{j}\right)\right) \\
& \rightarrow H^{0}\left(M, \vartheta\left(-C_{j}\right) / \vartheta\left(-C_{j}-E\right)\right) \simeq \mathrm{C} \rightarrow 0 .
\end{aligned}
$$

It follows readily that $\theta\left(-C_{j}\right) / \theta\left(-C_{j}-E\right)$ is a sheaf of germs of sections of a trivial line bundle over $\left(|E|, \theta_{E}\right)$ for $0 \leqslant j \leqslant l$.

Conversely, suppose $H^{0}\left(M, \theta_{C_{j}+E}\right) \rightarrow H^{0}\left(M, \theta_{C_{j}}\right)$ is surjective for $0 \leqslant j \leqslant$ $l$, and $\theta\left(-C_{j}\right) / \theta\left(-C_{j}-E\right)$ is the sheaf of germs of sections of a trivial line bundle over $\left(|E|, \theta_{E}\right)$ for $0 \leqslant j \leqslant l$. From (1.2) since $H^{0}\left(M, \vartheta\left(-C_{j}\right) / \vartheta\left(-C_{j}-E\right)\right) \simeq \mathbf{C}, \operatorname{dim} H^{0}\left(M, \theta_{C_{j}+E}\right)=\operatorname{dim} H^{0}\left(M, \theta_{C_{j}}\right)$ + 1. By induction, $\operatorname{dim} H^{0}\left(M, \theta_{C_{1}+E}\right)=l+1+\operatorname{dim} H^{0}\left(M, \theta_{Z}\right)=l+2$. However, $\operatorname{dim} H^{1}(M, \theta)=\operatorname{dim} H^{1}\left(M, \theta_{C_{l}+E}\right)=\operatorname{dim} H^{0}\left(M, \theta_{C_{l}+E}\right)=l+$ 2. So $p$ is a maximally elliptic singularity.

COROllary 2.5. Let $\pi: M \rightarrow V$ be the minimal good resolution of normal two-dimensional Stein spaces with $p$ as its only weakly elliptic singularity. Suppose $p$ is an almost minimally elliptic singularity but not a minimally elliptic singularity and $K^{\prime}$ exists. Then $v_{p}$ is Gorenstein if and only if $H^{0}(M, \mathcal{O}(-Z) / \mathcal{O}(-Z-E)) \simeq \mathbf{C}$.

3. Existence theorem for almost minimally quasi-simple elliptic singularities.

Definition 3.1. Let $\pi: M \rightarrow V$ be the minimal good resolution of a normal two-dimensional Stein space with $p$ as its only weakly elliptic singularity. If the minimally elliptic cycle $E=A_{1}$ is a nonsingular elliptic curve, we say that $p$ is a quasi-simple elliptic singularity.

THEOREM 3.2. Let $\pi: M \rightarrow V$ be the minimal good resolution of normal twodimensional Stein space with $p$ as its only quasi-simple elliptic singularity. Let $\Gamma$ denote the weighted dual graph along with the genera of the $A_{i}$. Suppose $K^{\prime}$ exists and $Z_{B_{0}}=Z, Z_{B_{1}}, \ldots, Z_{B_{l+1}}=Z_{E}=A_{1}$ is the elliptic sequence. Assume further that the coefficient of $A_{j}$ in $Z_{B_{i}}, 0 \leqslant i \leqslant l$, are equal whenever $A_{j} \cap A_{1}$ $\neq 0$ and $A_{j} \neq A_{1}$. Let $L_{j}$ be the line bundle corresponding to $\theta\left(-C_{j}\right) / \mathcal{O}\left(-C_{j}\right.$ $\left.-A_{1}\right), 0 \leqslant j \leqslant l$, over the elliptic curve $A_{1}$ where $C_{j}=\sum_{i=0}^{j} Z_{B_{i}}$. Then we can deform a suitably large infinitesimal neighborhood $B$ of the exceptional set of $p$ such that $L_{j}$ are trivial bundles over $A_{1}$ simultaneously and that $\Gamma$ is preserved. 
Proof. Let $Z_{B_{i}}=\sum C_{i, h} A_{h}$. Then $C_{0,1}=C_{1,1}=\cdots=C_{l, 1}=e_{1}=1$ by Corollary 2.6 of [30] where $E=e_{1} A_{1}$. For $0 \leqslant j \leqslant l, L_{j}$ are line bundles of Chern class zero over the elliptic curve $A_{1}$. Let $N$ be the normal bundle of $A_{1}$ in $M$. Let $Z_{B_{i}}=A_{1}+\sum_{j=2}^{n} C_{i, j} A_{j}+D_{i}$ where $A_{1}, \ldots, A_{n}$ are distinct, $A_{1} \cdot A_{j}$ $=1,2 \leqslant j \leqslant n$ and $D_{i}$ is a positive cycle which does not involve $A_{1}, \ldots, A_{n}$ and $A_{1} \cdot D_{i}=0$. Then $L_{j}=N^{-j} \xi_{p_{2}}^{-a_{2 j}} \ldots \xi_{p_{n}}^{-a_{n j}}$ where $P_{i}=A_{1} \cap A_{i}, a_{i, j}=$ $\sum_{h=0}^{j} C_{h, i}, 2 \leqslant i \leqslant n$, and $\xi_{p_{i}}$ are point bundles [7]. We want to show that by varying the point of intersection in $A_{1} \cap A_{i}, 2 \leqslant i \leqslant n$, we can vary $L_{j}$ in the Picard variety of $A_{1}$ and make $L_{j}$ trivial simultaneously for all $0 \leqslant j \leqslant l$. As $A_{1} \cdot Z_{B_{0}}=0$, we have $A_{i} A_{1}+C_{0,2}+\cdots+C_{0, n}=0$. We claim that there exists $n-1$ distinct points $q_{2}, \ldots, q_{n}$ such that $N^{-1}=\xi_{q_{2}}^{c_{0,2}} \cdots \xi_{q_{n}}^{c_{0, n}}$. In fact pick any fixed point $q \in A_{1}$, by Abel's theorem one can write

$$
N^{-1} \xi_{q}^{a_{1}}=\left(\xi_{q_{2}} \xi_{q}^{-1}\right)^{c_{0,2}} \cdots\left(\xi_{q_{n}} \xi_{q}^{-1}\right)^{c_{0, n}}
$$

for some $n-1$ distinct points $q_{2}, \ldots, q_{n}$ where $a_{1}=A_{1} \cdot A_{1}$. It follows easily that $N^{-1}=\xi_{q_{2}}^{c_{0,2}} \cdots \xi_{q_{n}}^{c_{0, n}}$. By our assumption $c_{0, i}=c_{1, i}=\cdots=c_{l, i}$ for $1 \leqslant$ $i \leqslant n$, hence $a_{i, j}=j c_{0, i}$

$$
\begin{aligned}
L_{j} & =N^{-j} \xi_{p_{2}}^{-a_{2 j}} \cdots \xi_{p_{n}}^{-a_{n j}} \\
& =\left(\xi_{q_{2}}^{c_{0,2}} \cdots \xi_{q_{n}}^{c_{0, n}}\right)^{j} \xi_{p_{2}}^{-a_{2, j}} \cdots \xi_{p_{n}}^{-a_{n, j}}=\left(\xi_{q_{2}} \xi_{p_{2}}^{-1}\right)^{a_{2, j}} \cdots\left(\xi_{q_{n}} \xi_{p_{n}}^{-1}\right)^{a_{n j}} .
\end{aligned}
$$

Now Abel's Theorem says that by varying the point of intersection in $A_{1} \cap A_{i}=\left\{P_{i}\right\}, 2 \leqslant i \leqslant n$, we can vary $\xi_{q_{i}} \xi_{p_{i}}^{-1}$ in the Picard variety of $A_{1}$ and make $\xi_{q_{i}} \xi_{p_{i}}^{-1}$ trivial so that $L_{j}$ is a trivial line bundle simultaneously for all $0 \leqslant j \leqslant l$.

COROLlary 3.3. The hypothesis is the same as Theorem 2.2. Then we can deform a suitable large infinitesimal neighborhood $B$ of the exceptional set such that $p$ is a Gorenstein singularity.

Proof. Trivial consequence of Theorems 2.3, 2.4 and 3.2.

4. Results on elliptic Gorenstein singularities with $H^{1}(M, \theta)=C^{3}$. We show in this section that for elliptic Gorenstein singularities with $H^{1}(M, \theta)=\mathrm{C}^{3}$, the elliptic sequence gives us a lot of information about the singularities.

Proposition 4.1. Let $\pi: M \rightarrow V$ be the minimal good resolution of normal two-dimensional Stein space with $p$ as its only weakly elliptic Gorenstein singularity. Let $A$ be the exceptional set. Suppose $H^{1}(A, Z)=0$ and the length of the elliptic sequence is three. Let $Z_{B_{0}}, Z_{B_{1}}, Z_{E}$ be the elliptic sequence. Let $D$ be the subvariety of $B_{1}$ consisting of those $A_{i} \subseteq B_{1}$ such that $A_{i} \cap|E|=\varnothing$. If $Z / D=Z_{B_{1}} / D$, then $\operatorname{dim} H^{1}(M, \theta)=3$, i.e. $p$ is a maximally elliptic singularity. 
Proof. By Theorem 3.9 of [30] $\operatorname{dim} H^{1}(M, \theta) \leqslant 3$. It is obvious that $p$ cannot be rational singularity or minimally elliptic singularity, i.e. $\operatorname{dim} H^{1}(M, \theta) \neq 0$ or 1 . By Theorem $\mathrm{C}$ of [30] $\operatorname{dim} H^{1}(M, \theta) \neq 2$. Therefore we have $\operatorname{dim} H^{1}(M, \theta)=3$.

TheOREM 4.2. Let $\pi: M \rightarrow V$ be the minimal good resolution of normal two-dimensional Stein space with $p$ as its only weakly elliptic Gorenstein singularity. Suppose $H^{1}(M, \theta)=C^{3}$ and $H^{1}(A, Z)=0$. Let $Z_{B_{0}}, Z_{B_{1}}, \ldots, Z_{B_{l}}$, $Z_{E}$ be the elliptic sequence. Let $D$ be the subvariety of $B_{l}$ consisting of those $A_{i} \subseteq B_{l}$ such that $A_{i} \cap|E| \neq \varnothing$. Suppose $Z / D=Z_{B_{l}} / D$. Then $m \theta \subseteq$ $\Theta\left(-\sum_{i=0}^{l-1} Z_{B_{i}}\right)$ and the multiplicity of the singularity is at least $-\sum_{i=0}^{l-1} Z_{B_{i}}^{2}$. If $Z_{E} \cdot Z_{E} \leqslant-2$, then $m \theta=\theta\left(-\sum_{i=0}^{l-1} Z_{B_{i}}\right)$. If $Z_{E} \cdot Z_{E} \leqslant-3$, then $\operatorname{dim} m^{n} / m^{n+1}=-n \sum_{i=0}^{l-1} Z_{B_{i}}^{2}$.

Proof. Look at the diagram (1.1). Since $p$ is a Gorenstein singularity and $\operatorname{dim} H^{1}\left(M, \Theta_{C_{l}+E}\right)=3$ (we denote $C_{j}=\sum_{i=0}^{j} Z_{B_{i}}$ ), we know that $H^{0}(M, \theta$ ) $\rightarrow H^{0}\left(M, \theta_{C_{l}}\right)$ is surjective and $\operatorname{dim} H^{0}\left(M, \theta_{C_{l}}\right)=2$. The five lemma asserts that $H^{0}\left(M, \vartheta\left(-C_{l}\right)\right) \rightarrow H^{0}(M, \vartheta(-Z))$ and $H^{0}\left(M, \vartheta\left(-C_{l}-E\right)\right) \rightarrow$ $H^{0}\left(M, \vartheta\left(-C_{l}\right)\right)$ are not the isomorphisms. There exist $f \in H^{0}\left(M, \vartheta\left(-C_{l}\right)\right)$ and $g \in H^{0}(M, \mathcal{O}(-Z))$ such that $f \notin H^{0}\left(M, \mathcal{O}\left(-C_{l}-E\right)\right)$ and $g \notin$ $H^{0}\left(M, \theta\left(-C_{l}\right)\right)$. We claim that $h \in H^{0}\left(M, \theta\left(-C_{l-1}\right)\right)$ for any $h \in$ $H^{0}(M, \theta(-Z))$ and $h \notin H^{0}\left(M, \vartheta\left(-C_{l}\right)\right)$. Since $m \theta \subseteq \theta(-Z)$, it will follow that $m \theta \subseteq \theta\left(-C_{l-1}\right)$. Also the multiplicity of ${ }_{v} \theta_{p}$ will be greater than or equal to $-C_{l-1}^{2}$ by Theorem 2.7 of [30]. There are two cases:

Case (i). Suppose $h \notin H^{0}\left(M, \vartheta\left(-C_{1}\right)\right)$. In the proof of Theorem 1.2 we know that

$$
\operatorname{dim} H^{0}\left(M, \theta_{C_{j+1}}\right)-1 \leqslant \operatorname{dim} H^{0}\left(M, \theta_{C_{j}}\right) \leqslant \operatorname{dim} H^{0}\left(M, \theta_{C_{j+1}}\right) .
$$

In fact, let $R_{j}$ be the image of $H^{0}\left(M, \theta_{C_{j+1}}\right) \rightarrow H^{0}\left(M, \theta_{C_{j}}\right)$, then

$$
\operatorname{dim} H^{0}\left(M, \theta_{C_{j+1}}\right)-1 \leqslant \operatorname{dim} R_{j} \leqslant \operatorname{dim} H^{0}\left(M, \theta_{C_{j+1}}\right) \text {. }
$$

The five lemma shows that $H^{0}(M, \theta) \rightarrow H^{0}\left(M, \theta_{C_{1}}\right)$ is surjective and $\operatorname{dim} H^{0}\left(M, \theta_{C_{1}}\right)=2$. Observe that $h^{j+1} \in H^{0}\left(M, \Theta\left(-C_{j}\right)\right)$ but $h^{j+1} \notin$ $H^{0}\left(M, \vartheta\left(-C_{j+1}\right)\right)$ for $0 \leqslant j \leqslant l$. So by induction, we have $H^{0}(M, \theta) \rightarrow$ $H^{0}\left(M, \theta_{C_{j+1}}\right)$ is surjective and $\operatorname{dim} H^{0}\left(M, \theta_{C_{j+1}}\right)=j+2$. In particular, $\operatorname{dim} H^{1}(M, \theta)=\operatorname{dim} H^{1}\left(M, \theta_{C_{l}+E}\right)=l+2$. Hence $p$ is a maximally elliptic singularity and $l=1$. Our claim holds trivially.

Case (ii). Suppose $\exists h \in H^{0}\left(M, \vartheta\left(-C_{1}\right)\right)$, and $h \notin H^{0}\left(M, \vartheta\left(-C_{l-1}\right)\right)$, then $l \geqslant 3$. Since $H^{1}(M, \theta)=C^{3}, \omega, f \omega, h \omega$ form a dual basis for $H_{*}^{1}(M, \vartheta)$ $\cong H^{0}(M \backslash A, \Omega) / H^{0}(M, \Omega)$ where * represents compact support and $\Omega$ denotes the sheaf of germs of holomorphic two forms and $f \in H^{0}(M, m \theta)$. By case (i) we may assume that $f \in H^{0}\left(M, \vartheta\left(-C_{l}\right)\right)$. Let $j$ be the least integer such that $f$ and $h$ are in $H^{0}\left(M, \vartheta\left(-C_{j}\right)\right)$. Then $1 \leqslant j \leqslant l-2$. Let $U$ be a 
suitably small holomorphically convex neighborhood of $\left|B_{j+1}\right|$ such that $f \omega$, $h \omega \in H^{0}\left(U \backslash\left|B_{j+1}\right|, \Omega\right)$ and one of $h \omega$ and $f \omega$ has no zeros on $U$ and such that $\Phi: U \rightarrow V_{1}$ represents $\left|B_{j+1}\right|$ as exceptional set with $V_{1}$ a normal two-dimensional Stein space. Let $p_{1}=\Phi\left(B_{j+1}\right)$. Then $p_{1}$ is a Gorenstein singularity and $\operatorname{dim} H^{1}(U, \theta) \geqslant 2$ because $f \omega, h \omega$ are linearly independent in $H^{0}\left(U \backslash\left|B_{j+1}\right|, \Omega\right) / H^{0}(U, \Omega) . Z_{B_{j+1}}, \ldots, Z_{B_{l}}, Z_{E}$ is the elliptic sequence of length $\geqslant 3$ relative to $\Phi$. Hence $\operatorname{dim} H^{1}(U, \theta)=\operatorname{dim} H^{1}\left(U, \theta_{C_{1}-C_{j}+E}\right)$ by Theorem 3.7 of [30]. The following exact sequence

$$
H^{1}\left(M, \theta_{C_{l}}\right) \rightarrow H^{1}\left(M, \theta_{C_{l}-C_{j}+E}\right) \rightarrow 0
$$

shows that $\operatorname{dim} H^{1}(U, \theta)=\operatorname{dim} H^{1}\left(M, \theta_{C_{l}-C_{j}+E}\right) \leqslant \operatorname{dim} H^{1}\left(M, \theta_{C_{l}}\right)=2$. Therefore $\operatorname{dim} H^{1}(U, \theta)=2$. But this is impossible because of Theorem $\mathrm{C}$ of [32].

Let $A_{i} \subseteq\left|B_{l}\right|$. We claim that $H^{0}\left(M, \theta\left(-C_{l-1}\right)\right) \rightarrow$ $H^{0}\left(M, \vartheta\left(-C_{l-1}\right) / \vartheta\left(-C_{l-1}-A_{i}\right)\right)$ is surjective. Otherwise $H^{0}\left(M, \vartheta\left(-C_{l-1}\right.\right.$ $\left.\left.-A_{i}\right)\right) \rightarrow H^{0}\left(M, \Theta\left(-C_{l-1}\right)\right)$ is an isomorphism. A similar argument of the proof of Theorem 1.1 will show that $H^{0}\left(M, \vartheta\left(-C_{l}\right)\right) \rightarrow H^{0}\left(M, \vartheta\left(-C_{l-1}\right)\right)$ is an isomorphism. By applying five lemma in diagram (1.1), we see that the image of $H^{0}(M, \theta)$ in $H^{0}\left(M, \theta_{C_{l}}\right)$ has dimension equal to one. This implies that $\operatorname{dim} H^{1}(M, \vartheta)=\operatorname{dim} H^{1}\left(M, \theta_{C_{1}+E}\right)=\operatorname{dim} H^{0}\left(M, \theta_{C_{1}+E}\right) \leqslant 2$, which is a contradiction. If $Z_{E} \cdot Z_{E} \leqslant-2$, then by the similar argument as the proof of Theorem D of [32], $m \theta=\theta\left(-C_{l-1}\right)$. The same argument as the proof of Theorem $\mathrm{D}$ of [32] also gives that $\operatorname{dim} m^{n} / m^{n+1}=-n \sum_{i=0}^{l-1} Z_{B_{i}}^{2}$ if $Z_{E} \circ Z_{E} \leqslant-3$.

COROLlaRy 4.3. The assumption is the same as Theorem 3.2. Suppose we assume further that $p$ is a hypersurface singularity. If $Z_{E} \circ Z_{E} \leqslant-1$, then $l \leqslant 4$. If $Z_{E} \circ Z_{E} \leqslant-2$, then $l \leqslant 2$. If $Z_{E} \circ Z_{E} \leqslant-3$, then $l=1$ (i.e. $p$ is $a$ maximally elliptic singularity), $m^{n}=H^{0}(A, \Theta(-n Z))$ and $\operatorname{dim} m^{n} / m^{n+1}=$ $-n Z \circ Z$.

Proof. Since $\operatorname{dim} H^{1}(M, \mathcal{\theta})=3$, by Theorem 1.3 of [31], multiplicity of $v_{p} \theta_{p}$ is less than or equal to 4 for hypersurface singularity. Recall that $-Z^{2} \geqslant-Z_{B_{1}}^{2} \geqslant \cdots \geqslant-Z_{B_{l}}^{2} \geqslant-Z_{E}^{2}$. Therefore $4 \geqslant-C_{l-1}^{2}=$ $-\sum_{i=0}^{l-1} Z_{B_{i}}^{2}$. The corollary follows easily.

\section{REFERENCES}

1. M. Artin, On isolated rational singularities of surfaces, Amer. J. Math. 88 (1966), 129-136.

2. H. Bass, On the ubiquity of Gorenstein rings, Math. Z. 82 (1963), 8-28.

3. H. Cohn, Some explicit resolutions of modular cusp singularities, Math. Ann. 198 (1972), 123-130.

4. P. DuVal, On isolated singularities of surfaces which do not affect the condition of adjunction, Proc. Cambridge Philos. Soc. 30 (1933/34), 453-491.

5. H. Grauert, Über Modifikationen und exzeptionnelle analytishe Mengen, Math. Ann. 146 (1962), 331-368. 
6. H. Grauert and $\mathrm{O}$. Riemenschneider, Verschwindungssätze für analytische Kohomologiegruppen auf Komplexen Räumen, Invent. Math. 11 (1970), 263-292.

7. R. Gunning, Lectures on Riemann surfaces, Princeton Univ. Press, Princeton, N. J., 1966.

8. R. Gunning and H. Rossi, Analytic functions of several complex variables, Prentice-Hall, Englewood Cliffs, N. J., 1965.

9. F. Hirzebruch, The Hilbert modular group, resolution of singularities at the cusps and related problems, Séminaire Bourbaki, 1970/71, Exposé. 396.

10. F. Hirzebruch, W. Neumann and S. Koh, Differentiable manifolds and quadratic forms, Lecture Notes in Pure and Appl. Math., vol. 4, Dekker, New York, 1971.

11. U. Karras, Dissertation, Univ. of Bonn, 1973.

12. __ Eigenshaften der lokalen Ringe in zweidimensionaten Spitzen, Math. Ann. 215 (1975), 117-129.

13. D. Kirby, The structure of an isolated multiple point of a surface. I, Proc. London Math. Soc. 6 (1956), 597-609.

14. _ The structure of an isolated multiple point of a surface. II, Proc. London Math. Soc. 7 (1957), 1-28.

15. H. Laufer, Normal two-dimensional singularities, Ann. of Math. Studies, no. 71, Princeton Univ. Press, Princeton, N. J., 1971.

16. On rational singularities, Amer. J. Math. 94 (1972), 597-608.

17. _ Deformations of resolution of two-dimensional singularities, Rice Univ. Studies 59

(1973), Vol. 1, 53-96.

18. __ Taut two-dimensional singularities, Math. Ann. 205 (1973), 131-164.

19. __ On minimally elliptic singularities, Amer. J. Math. 99 (1977), 1257-1295.

20. J. Lipman, Rational singularities with applications to algebraic surfaces and unique factorization, Inst. Hautes Etudes Sci. Publ. Math. 36 (1969), 195-279.

21. D. Mumford, The topology of normal singularities of an algebraic surface and a criterion for simplicity, Inst. Hautes Etudes Sci. Publ. Math. 9 (1961), 5-22.

22. P. Orlik and P. Wagreich, Isolated singularities of algebraic surfaces with $\mathbf{C}^{*}$ action, Ann. of Math. (2) 93 (1971), 205-228.

23.

24. K. Saito, Einfach-elliptische Singularitaten, Invent. Math. 23 (1974), 289-325.

25. Y.-T. Siu, Analytic sheaf cohomology groups of dimension $\boldsymbol{n}$ of $\boldsymbol{n}$-dimensional complex spaces, Trans. Amer. Math. Soc. 143 (1969), 77-94.

26. G. Tyurina, Absolute isolatedness of rational singularities and triple rational points, Functional Anal. Appl. 2 (1968), 324-332.

27. P. Wagreich, Elliptic singularities of surfaces, Amer. J. Math. 92 (1970), 419-454.

28. __ Singularities of complex surfaces with solvable local fundamental group, Topology 11 (1972), 51-72.

29. J. Wahl, Equisingular deformations of plane algebroid curves, Trans. Amer. Math. Soc. 193 (1974), 143-170.

30. Stephen S.-T. Yau, On maximally elliptic singularities, Trans. Amer. Math. Soc. (to appear).

31. _ Hypersurface weighted dual graphs of normal singularities of surfaces, Amer. J. Math. (to appear).

32. __ On almost minimally elliptic singularities, Amer. J. Math. (to appear).

33. _ On strongly elliptic singularities, Amer. J. Math. (to appear).

34. O. Zariski, Studies in equisingularity. I, Equivalent singularities of plane algebroid curves, Amer. J. Math. 87 (1965), 507-536. 08540

Department of Mathematics, Institute for advanced Study, Princeton, New Jersey

Department of Mathematics, Harvard University, Cambridge, Massachusetts 02138 (Current address) 